mais se rétablit très rapidement. On ne peut, en effet, dans la fabrication à la ferme, envisager une pasteurisation des crèmes, suivie d'un ensemencement avec des ferments lactiques purs, comme cela se pratique dans les usines américaines.

Le seul procédé qui nous ait donné quelques résultats, avec les crèmes rances au moment du barattage, est celui qui consiste à faire un grain de beurre très petit (gros comme la moitié d'un grain de blé). On le lave ensuite 4 ou 5 fois dans la baratte, en veillant à ce que le grain ne grossisse pas pendant les lavages. Les premières eaux ont une odeur rance accentuée que n'ont plus les dernières. Le beurre n'est pas mauvais mais il ne se conserve pas beaucoup plus de 24 heures.

\title{
REFLEXIONS SUR L'EMPLOI DU FROID EN LAITERIE
}

Par J. BLIER,

Docteur-Vétérinaire

Qu'est-ce que le froid? Et qu'est-ce que la chaleur? On ne le sait pas, et les physiciens l'avouent. Pourtant de plus en plus les industriels du lait font appel à l'appareil qui "refroidit»; depuis plus longtemps encore ils usent d'appareils à " chauffer ". Cela démontre, une fois de plus, que l'emploi d'une technique laitière précède en général son explication. Cela prouve aussi l'intérêt des examens de conscience à pratiquer dans les congrès.

En effet, les congrès ne peuvent guère avoir un rôle utile que s'ils permettent l'inventaire sincère des questions à l'ordre du jour pour les techniciens et surtout que s'ils aiguillent les praticiens et les chercheurs vers des trouvailles.

Tentons cet examen de conscience, cet inventaire, et d'emblée fixons un premier point au sujet duquel l'accord est unanime.

I. Pour que l'usage du froid, dans l'industrie laitière, ait sa raison d'être, il faut que quatre conditions soient remplies, à savoir : que cet usage soit hygiénique, utile, financièrement viable, et que le but visé ne puisse être atteint par un autre procédé meilleur marché que le froid.

Hors du respect de ces quatre conditions, on est assuré d'un échec.

Rappeler ce postulatum s'impose, car l'industrie du froid est pavée, surtout à propos du lait, de trop de légendes peut-être entretenues.

Il faut ici les dissiper, et regarder si l'industrie laitière possède l'instrument propre à l'étude de l'utilisation du froid. C'est ce que nous allons voir ici-même.

(1) Cê travail a été présenté en rapport au dernier Congrès international de laiterie. 
Vers L'inventatre. - Pour l'observateur impartial, un contraste frappant éclate en effet depuis longtemps, entre les apologistes du froid et la prudence des industriels à l'utiliser. Ceux-là parlent toujours de l'incompréhension dont l'œuvre de Michel TeLtien, le père du froid, est l'objet, tandis que ceux-ei qui savent établir un bilan, et veulent bien être des adeptes du froid s'il leur est indispensable, n'ont pourtant qu'un enthousiasme limité pour la machine à froid. Pourquoi ? Parce que cette machine est onéreuse.

Il faut, par exemple, dépenser environ 10 ealories pour obtenir une frigorie. Il faut, par exemple, comparer la machine à froid, opérant dans une enceinte, à une pompe en marche dans un navire erevé d'une voie d'eau puisque aucun dispositif connu n'est étanche aux échanges thermiques, et que, comme le navire menacé, le frigorifique a de tous côtés des entrées en calories.

$\mathrm{Au}$ contraire, tous les autres procédés de conservation des denrées périssables sont moins coûteux. L'anarchie, qui règne dans les sciences de l'alimentation, n'a pas encore permis d'introduire, pour tous les modes de lutte contre les microbes, une commune unité de mesure.

L'épicier, qui conserve sa morue salée, poursuit pourtant le même objectif que le fabricant de boittes de thon ou de fromage.

Or, nous n'avons, dans l'état actuel des choses, aucun élément de comparaison entre les rendements propres à chaque technique de conservation, il n'y a même pas de liaison entre les diverses industries alimentaires. On étonne un sardinier de Lorient quand on lui parle des autoclaves agitateurs, utilisés depuis longtemps pour la fabrication des laits stérilisés.

De ce chocs se dégagent ces deux faits, trop méconnus des théoriciens, et saisissants pour les industriels: a) le froid artificiel est cher; b) la chaleur conserve à bon marché.

Et pourquoi, malgré cela, le froid a-t-il un si grand intérêt? Parce qu'il est le seul antimicrobien qui ne tue pas. En dehors des autres rôles que l'on pourrait, comme nous le verrons, lui attribuer, car il y en a d'autres, il sera donc, par-dessus tout et avant tout, l'auxiliaire de la pasteurisation et l'indispensable allié du lait cru que l'on tient à garder "vivant»; il intervient ici dans des conditions qui ne stérilisent pas. C'est là le privilège de l'appareil frigorifique en laiterie. Et comme, sous les efforts des biologistes, l'avenir est à l'aliment qui vit - adversaire victorieux de l'aliment mort - et que seul le froid ne tue pas la denrée conservée, il faut bien, de gré ou de force, faire appel au froid.

Pour connaître, dans l'industrie laitière, toutes les applications actuelles du froid, un inventaire patient de ses utilisations, réalisées ou possibles, s'impose. Je les résume, pour gagner de la place, dans le tableau suivant : 


\section{L'INVENTAIRE}

Modes divers de l'usage du froid en laiterie.

Rôle antimicrobien. - En général le seul qu'on lui attribue; or, c'est inexact. Usage culinaire. - Industrie des Sorbets. La formidable industrie de l'icecream aux Etats-Unis.

Action solidificatrice. - Emploi de cette action en margarinerie, en beurrerie, dans l'industrie du "lait reconstitué ".

Rôle en fromagerie. - Action accélératrice dans l'emprésurage par contact préalable à froid du lait et de la présure,- - Caves de maturation du Roquefort.

Perfectionnement des ateliers. - Rôle antibueés; constance du degré hygrométrique en fromagerie; captage des poussières.

Emploi au laboratoire de recherches.- Etude des germes cryophiles; cryoscopie; étude du point de congélation des corps gras; conservation aux fins d'analyse.

Rôle de séchage, soit :

a) Par élimination mécanique de glaçons présents dans du lait congelé un peu au-dessous du point cryoscopique ;

b) Par élimination mécanique de glaçons présents dans le lait brusquement amené à son point cryohydratique global (dans ce cas, l'eau à éliminer serait chimiquement pure) ;

c) Soit par les procédés de séchage employés actuellement avec succès dans l'industrie opothérapique, grâce à de l'air totalement déshydraté par givrage.

Voilà ce que nous donne l'inventaire des emplois, actuellement connus, du froid en laiterie.

On comprendra, devant leur nombre déjà imposant, notre briéveté à les examiner un par un.

$1^{0}$ Le froid stabilisateur antimicrobien. - Pourquoi cette expres. sion? Parce que, exception faite des germes cryophiles, dont l'étude, amorcée par les travaux de BIDAULT, vétérinaire principal de l'armée et par quelques chercheurs, s'annonce très vaste, le froid entrave la pullulation des microbes thermophiles, mais ne les détruit pas. Tout ce qu'il peut faire - et trop souvent on l'oublie - c'est de les stabiliser.

Cette stabilisation varie avec chaque microbe, avec la quantité de lait, son épaisseur, la durée d'action du froid, l'action de la lumière, l'épaisseur de la couche de crème (facteur d'anaérobiose), la rapidité des échanges thermiques, la présence ou l'absence de spores, la mobilité du lait, la nature des surfaces de contact et bien d'autres facteurs, dont chacun justifierait une étude précise à poursuivre dans les laboratoires spéciaux, mais qui tous nous permettent de conclure dès maintenant qu'avec le froid stabilisateur antimicrobien, on est toujours en présence de cas d'espèce, à examiner un par un, et qu'aucune loi générale ne peut être formulée à leur propos, sauf celle-ci : pour être efficace, comme agent de conservation, le froid doit respecter l'équilibre des élé- 
ments du lait. Employé comme facteur de longue conservation, il échoue en effet, et toutes les tentatives poursuivies en ce sens n'ont pas abouti contrairement à ce qui se passe pour la viande frigorifiée ou même pour les beurres et les fromages qui s'accommodent de longs séjours audessous de zéro degré centigrade dans les entrepôts frigorifiques.

Des études bactériologiques seraient nécessaires pour étudier, dans chacun de ces cas d'espèce, avec le concours de physiciens, l'action antimicrobienne et stabilisatrice du froid sur le lait. Donnons un seul exemple de la difficulté des questions à envisager. Pour éviter à un lait pasteurisé, brusquement refroidi par passage sur un réfrigérateur ondulé, parcouru lui-même par de l'eau froide et un fluide frigorifique, le goût de cuit, il faut, comme l'a montré J. LưCAs, mettre ce lait au contact d'un air lui-même dépoussiéré par passage sur un système frigorifique capable, par givrage, de capter son humidité et ses poussières.

Ce que nous avons dit de la cherté du froid artificiel nous amène à indiquer ici le grand intérêt de la recherche systématique des eaux froides en laiterie, même par forage. C'est là encore un cas d'espèce à soumettre à une discussion d'ordre mathématique, dans chaque installation.

Quant aux microbes cryophiles, pour montrer l'intérêt de leur étude, trop négligée, signalons ce seul fait, découvert par GoRINI, d'un germe capable à la température de $+12^{\circ}$ d'attaquer la caséine et, au contraire, à $+35^{\circ}$ de donner de l'acide lactique avec le lactose. Voilà done un microbe qui fait volte-face suivant la température. C'est prouver, de plus, d'une façon saisissante qu'en bactériologie, toute for mule d'ensemble n'est point viable; il y a des microbes qui vivent à $+3^{\circ}$, ne l'oublions point.

Des dispositifs mécaniques très variés favorisent sur le lait cette action stabilisatrice du froid. On connaît les modèles de réfrigérants ondulés qui constituent, en définitive, la part la plus imposante de l'arsenal frigorifique laitier actuel; nous n'avons pas à les décrire et à discuter de leurs diverses formes.

$\mathrm{Au}$ rôle antimicrobien stabilisateur du froid se rattachent diverses questions d'un vif intérêt, notamment:

$1^{\circ}$ La production, le transport et la vente du lait cru, propre, sain et vivant conformément au programme de la Ligue du Lait ou d'autres groupements analogues qui opèrent à l'étranger ;

$2^{\circ}$ Le transport des laits refroidis avec les divers types de récipients, depuis le camion frigorifique automobile, récemment créé à Rio de Janeiro, par Rocha, jusqu'au vagon-cuve isotherme lequel m'apparaît. par l'économie de main-d'œuvre, la solution d'avenir, sur les voies ferrées. 
$3^{0}$ Une grande partie du problème de l'urbanisme du lait, lui-même, dominé essentiellement par ce point trop négligé, de l'intérêt du refroidissement précoce en matière de laiterie; e'est à la porte de la vacherie elle-même, sinon dans la vacherie que le froid doit intervenir d'abord sur le lait. Ce liquide rencontrera ou devrait rencontrer ensuite sur sa route le froid, non seulement à toutes ses étapes, depuis le départ, mais aussi à son point d'arrivée dans la halle frigorifique au lait.

Seule, en effet, la halle frigorifique au lait, combinée avec le transport frigorifique, permettrait, dans les grandes villes, l'arrivée du lait à tout moment, rendrait possible, par un stage d'environ vingt-quatre heures, l'examen du lait par la réaction de l'alcool-alizarine ou de la catalase, sans préjuger des autres moyens de contrôle. On allégerait ainsi tout en étendant les limites des bassins laitiers, la dure journée du garçon livreur obligé d'aller charger, avant 2 heures du matin dans les gares, à Paris, un lait trait la veille qu'il livre en toute hâte aux détaillants, qui ont eux-mêmes à le répartir entre les consommateurs de 6 à 9 heures du matin.

Le lait ne souffrirait nullement d'un séjour d'une journée dans un frigorifique, à une température pas au-dessous de $0^{\circ} \mathrm{C}$. ou très voisine $\left(0^{\circ}\right.$ à $4^{\circ} \mathrm{C}$.). Les halles frigorifiques pourraient être dans les gares.

Tels sont, à grands traits, les chapitres cardinaux de l'histoire du froid en laiterie envisagé comme stabilisateur antimicrobien.

On voit : $1^{\circ}$ qu'il n'est pas un améliorateur; $2^{\circ}$ même qu'il favorise l'action des germes cryophiles sur lesquels nous ne savons presque rien ; $3^{\circ}$ mais qu'il a l'avantage de paralyser les actions microbiennes capables soit par la coagulation acide, soit même par le jeu des organismes thermophiles, d'altérer le lait. Il évite des désordres alarmants, mais ne peut jamais rendre mieux que ce qu'on lui a donné. Avee le froid on ne retrouve jamais que ce qu'on lui apporte.

C'est là le fait qui domine son histoire.

$$
*^{*} *
$$

$2^{\circ}$ Poursuivant notre enquête, nous trouvons, en France, en Angleterre, en Suisse, etc., mais surtout aux Etats-Unis, le froid, jouant, avec le lait, un rôle culinaire. Il faut avoir entrevu ce qu'est la gigantesque industrie de l'Ice-Cream pour saisir tout l'intérêt de cet aspect de l'industrie frigorifique. Nous n'avons qu'à renvoyer le lecteur à la revue Ice-Cream and Refrigeration pour qu'il prenne contact avec une industrie qui occupe, sur le territoire de l'Union, des milliers de personnes et dont l'extension augmente, d'une façon prodigieuse,la consommation du lait et celle des produits accessoires tels que la gélatine, les colorants et les parfums. L'industrie de la crème glacée, véritable sorbet, aux Etats-Unis dépasse, en importance, l'industrie des fro- 
mages chez nous. Et la mentionner ici s'impose pour nous habituer à voir, dans l'emploi du froid en laiterie, autre chose que son simple rôle antimicrobien.

L'apparition en France des sorbets au chocolat, vendus dans les salles de cinématographe, sous les noms de "Nanouk ", d' "Eskimos ", etc., marque, pour nous, les débuts de ce débouché pour l'industrie laitière.

$$
*^{*} *
$$

$3^{\circ}$ Nous rencontrons maintenant sur notre route le froid solidificateur, employé notamment en oléo-margarinerie pour favoriser la prise en masse des émulsions et augmenter leur consistance. On peut, par exemple, pétrir des produits fusibles aux températures ordinaires sur une table ou mieux dans un carter refroidi, par la circulation d'un courant de saumure et à l'abri des poussières. On connaît le rôle du. froid en beurrerie, non seulement pour l'entreposage, l'interruption de la maturation de la crème, mais aussi dans le barattage pour la diminution de la richesse en eau.

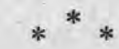

$4^{\circ}$ Nous rencontrons l'appareil frigorifique, en laiterie, dans certains ateliers, pour régler, par le givrage, l'état hygrométrique de l'air, pour capter les buées et les poussières incluses dans les gouttelettes.

$5^{\circ}$ Nous le trouvons également dans l'industrie peu connue du lait reconstitué; il intervient, en effet, en facilitant le transport de la crème congelée que l'on émulsionne au lieu de fabrication, dans des solutions, réalisées ensuite sur place, de lait en poudre.

$6^{\circ}$ On connaît l'action du froid sur la présure. Celle-ci ne coagule point le lait refroidi, mais mise préalablement à son contact, elle accélère la coagulation du lait dès que celui-ci se trouve à nouveau à une température favorable. Il y a là un moyen pour abréger les emprésurages. Toujours en fromagerie, on connaît le rôle de la constance isothermique des caves de Roquefort.

$7^{\circ}$ Nous trouvons maintenant le froid auxiliaire $d u$ chimiste:

D'abord par son intervention dans les recherches cryoscopiques, puis par son rôle, que malheureusement on a abandonné, comme conservateur du lait en vue de l'analyse.

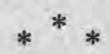

Mais l'inventaire systématique nous révèle des surprises. Si l'on examine en effet toutes les ressources du froid, nous apercevons des applications inexplorées.

Le succès du procédé OTTESEN dans les industries de la pêche, basé sur l'emploi des propriétés du point cryohydratique devrait attirer 
l'attention dans l'industrie laitière. Aux températures cryohydratiques, l'eau se libère entièrement des corps qu'elle tenait dissous et devient chimiquement pure.

Nous ne connaissons rien du point eryohydratique global du lait qu'il faudrait déterminer pour chacun de ses constituants.

Quel est, par exemple, le point cryohydratique d'une solution de lactose, d'une solution de easéinate? Les tentatives de congélation du lait réalisées en vue des transports à longue distance ne sont jamais entrées, malgré des efforts déjà anciens, dans la pratique courante, mais peut-être n'a-t-on jamais visé à obtenir, à des températures très basses, aux environs de $-22^{\circ}$, des congélations brusques du lait analogues aux congélations du procédé OTTESEN qui sont en train de révolutionner le transport frigorifique du poisson.

Il y a là une propriété qui mérite une étude approfondie, car elle s'annonce eapable de libérer, à l'état de pureté chimique, l'eau du lait sans faire subir à ce dernier l'action de la chaleur, donc en respectant tout ce qu'il contient (1).

On n'a jamais, à notre connaissance, employé dans l'industrie laitière les procédés frigorifiques utilisés avec tant de succès en opothérapie.

On voit que l'inventaire que nous venons d'effectuer donne de riches résultats et que dans les futurs congrès de l'industrie laitière plusieurs rapports, en réalité, devront être consacrés aux applications, déjà si nombreuses, du froid à l'industrie laitière.

Le froid mérite donc d'être étudié par les savants du lait.

$$
*^{*} *
$$

Les chercheurs de la question du lait, peuvent-ils étudier le lait? Il est inutile de dire que l'étude de eette question est justifiée; mais peut-on la poursuivre comme il conviendrait? Difficilement, faute d'outillage. Prenons d'abord quelques exemples :

C'est d'une façon toute fortuite qu'un ingénieur français a inventé des accumulateurs de froid qui permettent aux automobilistes, le dimanche, d'emporter de Paris des sorbets qu'ils mangent à 200 kilomètres du point de départ; mais nous ne savons toujours pas, par exemple, quelles modifications un séjour de longue durée peut imposer à la crème glacée placée dans ces accumulateurs. Tout le monde dans l'industrie laitière est partisan de l'́mploi de l'€au glacée, soit naturelle,

(1) L'étude soignée du point eryohydratique des solutions serait partieulièrement utile pour la fabrication de la présure. La concentration par le froid des solutions de présure est à priori souhaitable, mąis il ne semble pas que l'industrie dans son ensemble, $s^{\prime} y$ soit appliquée. Si l'on aboutissait dans cette voie, une modification de la technique habituellement suivie s'jmposerait (Ch. Porcher). 
soit même artificielle, pour la réfrigération. Rien n'empêche, dans l'appareil frigorifique à compression, que je n'ai pas à décrire, de remplacer la saumure des bacs à immersion, habituellement incongelable, par de l'eau ordinaire qui se congèlerait en formant un manchon autour des tuyaux à détente directe. Ce manchon de glace, une fois la machine arrêtée pendant les heures de travail du lait, pourrait, à son tour, être recouvert d'eau courante qui se glacerait à son contact et qui serait envoyée sur le circuit du réfrigérant ondulé. L'avantage de ce dispositif, dû à l'ingénieur Marius Brossé, est le suivant : $i l$ permet de faire marcher la machine frigorifique aux moments perdus où l'on n'a pas à se préoccuper du travail du lait, ce qui permet de récupérer le travail du mécanicienfrigoriste, ainsi libéré lors de la manipulation du lait.

Le professeur Ch. Porcher, avee raison, a noté le gaspillage de glace aux Etats-Unis, glace naturelle récoltée pendant l'hiver et conservée pour l'été dans des silos. Que fait-on en France pour organiser, au moins dans les montagnes ou dans l'Est, la récolte de la glace naturelle produite pendant l'hiver? Absolument rien.

Le Danemark a conquis, grâce à l'emploi du froid, une grande partie du marché anglais avec ses beurres. Or, on s'obstine encore en France à fabriquer des beurres fermiers; il y en a d'excellents. mais la régularité de leur qualité est plutôt l'exception.

La puériculture devrait être en liaison étroite avec l'industrie du froid pour étudier la conservation à la maison, en récipient convenable, du lait refroidi des petits enfants; le professeur Ch. Porcher réclame depuis des années le récipient modèle qui permettra de le faire et il propose la caisse légère dans la double paroi de laquelle on ferait le vide.

Toute la bactériologie frigorifique, pour la conservation des beurres en entrepôts, devrait pouvoir être approfondie germe par germe et ce n'est pas là une mince besogne.

On oublie, sans cesse, qu'il y a une topographie de la bactériologie laitière et que tout résultat, bactériologiquement valable à tel endroit, peut différer assez nettement à tel autre et qu'il faudrait d'abord, pour la laiterie entière d'un pays donné, dresser la carte topographique, non seulement de la flore laitière, "mais aussi de la flore mammaire. Les fromages rapportent assez dans tous les pays du monde pour qu'on se permette ce travail utile.

Nous n'avons pas de travaux sur l'influénce des mouvements imprimés au lait pour sa conversation. Or, LUCET a montré depuis longtemps le rôle favorisant de l'agitation sur le développement d'une culture,

LuCAS a montré - et c'est très importañt - qu'un lait refroidi exposé à l'air se souille plus vite qu'un lait ordinaire puisqu'en vertu 
du principe de la paroi froide, il s'enrichit des germes apportés par la buée qui se dépose sur lui.

Hammer et JoHnson ont montré que la chaleur spécifique du lait n'était pas toujours la même (1). Le conservation du lait par des blocs de lait congelé, pour les transports à longues distances, ne s'est pas répandue et ne semble pas, malgré la tentative danoise du procédé CASSE (2) qui remonte à 1896, reposer sur des bases pratiques.

On a essayé de fabriquer de la neige de lait qu'on a ensuite essorée pour obtenir un liquide dans lequel les éléments du lait seraient très concentrés; ce serait le premier stade de la dessiccation. Ces tentatives ont été abandonnées.

On n'a pas étudié l'influence des surfaces des récipients sur la propagation du froid à travers le lait, ni leur aptitude au nettoyage. Toute la question du bidon isolant pour lait refroidi est à aborder. L'ingénieur RochA, à Rio de Janeiro, emploie, avec succès pour la conservation du lait refroidi, livré dans Rio par des camions-citernes frigorifiques, le givre considéré par lui comme un sous-produit utilisable de sa fabrique de glace. Du dégivrage envisagé par beaucoup de frigoristes comme une dépense, il en fait un profit.

\section{** *}

Mille problèmes analogues se poseraient à propos de l'action de la chaleur sur le lait dont je ne parlerai point et qui est d'ailleurs beaucoup mieux connue, car elle est étudiée depuis beaucoup plus longtemps.

Pas plus pour la chaleur que pour le froid, pas plus que pour tous les autres problèmes, les industriels du lait ne disposent d'aucun instrument de recherches. Chacun d'eux isolément, et avec raison, recule avant de lancer un technicien, lui-même isolé, sur le sentier onéreux des essais de laboratoire, parfois chimériques, toujours aléatoires, rarement récompensés par un bénéfice, qui se fait d'ailleurs toujours attendre, même quand il se produit.

L'industriel a raison et le vent n'est pas aux gaspillages.

Pour munir les chercheurs de l'instrument de travail nécessaire, une vaste coordination de l'industrie laitière, s'impose avee un sacrifice léger pour chacun, auquel devrait d'ailleurs, en toute justice, participer le public des consammateurs. Il faut doter l'industrie laitière d'un Institut du lait, e'est-à-dire d'un établissement scientifique capable de recherches de longue haleine poursuivies sans l'espoir d'aucun bénéfice matériel. C'est sur ce dernier point que j'insiste.

(1) Voir la revue le Lait, no 10, année 1922.

(2) Voir : Professeur Ch. Poncher, Hygiène de la viande et du lait, t. IV, p. 217, 1920. 